\title{
VARIABILITY IN ACTIVE GALACTIC NUCLEI
}

\author{
M. MATSLOKA \\ Institute of Physical and Chemical Research (RIKEN), \\ Hirosawa, Wako-shi, Saitama 351-01, Japan
}

\begin{abstract}
AGN (Active Galactic Nuclei) have their profound time variability over a wide range of time scales. Although many results of $A G N$ variability have been provided from wide band wavelength observations, I would like to concentrate the recent problems concerning a nearby region of their central engine based on the X-ray observations which are most efficient to investigate this region. In this paper we will investigate mainly the result of Seyfert galaxies which would be generalized to other AGN.
\end{abstract}

\section{Introduction}

Various variabilities of AGN have given much information to investigate the structure of AGN. Prior to description of the variability in AGN I would like to introduce a recent progress of the structure of AGN which has been obtained from spectrum observations as well as time variabilities.

A schematic overall spectrum of AGN is given by Sanders et al. (1989) who have derived from their investigations of wide band continuum observations of radio to X-ray for more than $100 \mathrm{AGN}$. The radio is emitted by non-thermal high energy electrons produced often in relation to a jet-like non-thermal phenomenon in AGN. It has been well known that AGN have generally BLR (broad line region) and NLR (narrow line region) in optical emissions (e.g. Netzer et al. 1990). In 1980 era remarkable UV bump was discovered by IUE observations (Malkan and Sargent 1982). This UV bump is represented by a black body spectrum with a temperature of several tens times $10^{3} \mathrm{~K}$. On the other hand, it has been known that in X-ray region overall continuum spectra of $A G N$ are represented by a power law function.

Recently an evidence of cold thick gas, so called "accretion or molecular torus" with $\mathrm{H}_{2}$-molecule is pointed out by IR $\mathrm{H}_{2}$-line $(\sim 2 \mu)$ observations (Kawara et al. 1989; Kawara et al. 1990). This accretion torus would reflect somewhat covering thick matter related to a discovery of polarized broad line emissions in the Seyfert 2 galaxy NGC1068 (Antonucci and Miller 1985) and very thick absorption gas in Seyfert 2 galaxies made by $\mathrm{X}$-ray observations (Koyama et al. 1989; Awaki et al. 1990). Consequently, the unified model of Seyfert $1 \& 2$ has been emphasized (Miller and Goodrich 1990), but the unification of AGN can not be so simply accepted (Mulchaey $\epsilon t$ al. 1992).

On the other hand a theoretical investigation has been in favour of an accretion disk surrounded a black hole. So called "UV bump" is considered to be related to such an accretion disk (Malkan and Sargent 1982; Sun and Malkan 1989), while recently Ginga discovered an X-ray hard bump above $10 \mathrm{keV}$ (Matsuoka et al. 1990; Pounds et al. 1990; Piro et al. 1990), which 

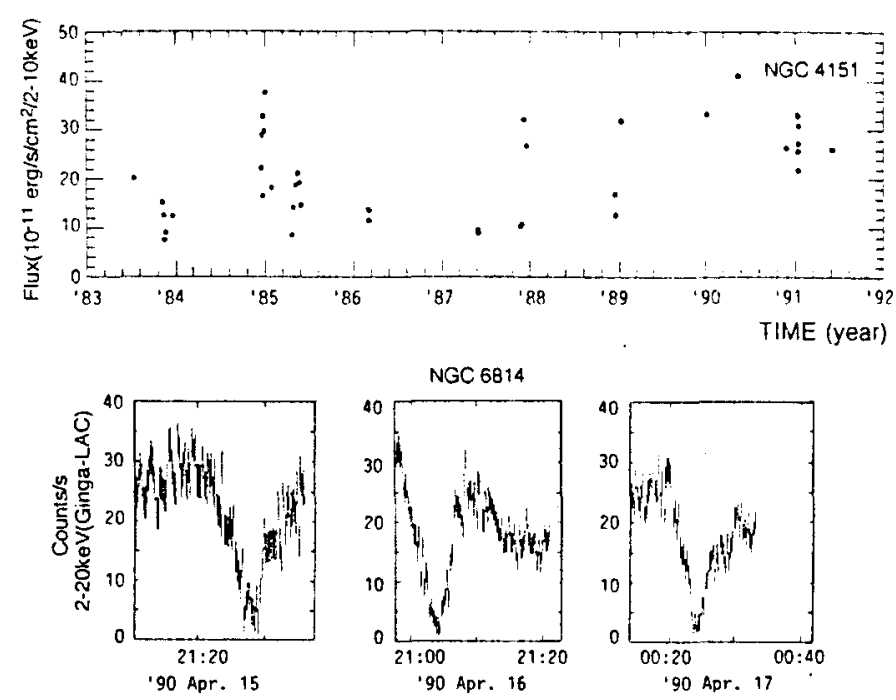

Fig. 1. X-ray light curves of NGC4151 (Yaqoob et al. 1992) and NGC6814 (Kunieda et al. 1991).

probably is related to this accretion disk or UV bump. Consequently it has been considered that general X-ray spectra of AGN exhibit a composite spectrum consisting of the direct and reflection components (Lightman and White 1988; Guilbert and Rees 1988).

In the following I would like to mention about model structures and $\mathrm{X}$ ray variabilities near the AGN central engine. It is noted that most figures in this paper are modified from the original ones without essential change.

\section{Variabilities of AGN}

\subsection{Continuum Variability}

Firstly I would like to show several snaps of X-ray variabilities of AGN. Fig. 1 are examples of the light curves in two Seyfert galaxies which show a long term X-ray variability of NGC4151 (Yaqoob et al. 1992) and a short term $\mathrm{X}$-ray variability of NGC6814 (Kunieda et al. 1991). As shown in this figure the time variabilities are seen over several years to several tens sec. Fig. 2 shows the X-ray variabilities from the AGN which are observed by EXOSAT. A Scale of 10 hour-time is shown in each figure (Pounds and McHardy 1990; McHardy 1989).

When we performed a standard power spectrum density (PSD) analysis, we obtained that these AGN gave a significant power suggesting a rapid variability as shown in Fig. 3 . The PSD is extended to a frequency of $10^{-2}$ $\mathrm{Hz}$ or higher. Generally we can describe the X-ray PSD of AGN as a function 

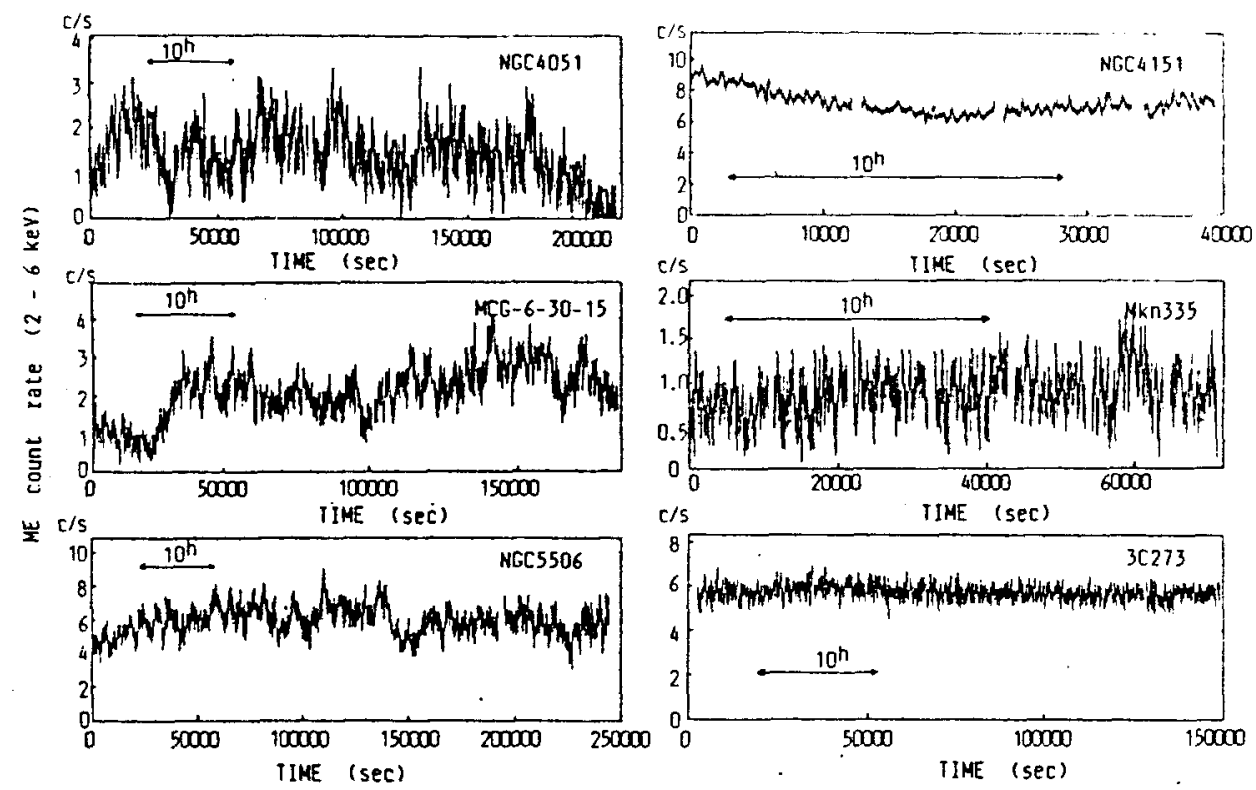

Fig. 2. Examples of X-ray light curves observed by EXOSAT (Pounds and McHardy 1988).

of $f^{-\alpha}$ for frequency $f$. The value of $\alpha$ is about $1 \sim 2$ for high frequencies and then decreases to be flat towards low frequencies.

Mathematical ways of achieving this PSD are considered (Begelman and De Kool 1991); e.g. (1) one way in which a Poisson process can produce $f^{-1}$ noise is to have a pulse shape with a very slow decay, (2) a second way to obtain a steeper power law is to superpose white noise spectra with a range of normalizations and high-frequency cut offs, and (3) a third way is to assume that there are correlations between the pulse height and either the decay time of the pulse or the mean rate of pulses. In any case the PSD of AGN X-ray intensity would reflect a chaotic central phenomenon of AGN. A simple interpretation of producing spectrally invariant intensity variations is released of stored energy in electron pairs or magnetic fields by shocks, but neither simple shot noise models nor models dominated by a simple cooling or heating time scale. However, the real physical activity in this most inside region is open to question.

\subsection{Iron Line Variability}

Next we will consider some outside region away from the central engine. The K X-ray iron line from Seyfert galaxies, one of major groups of AGN, generally has been known to be produced by the fluorescent mechanism in the cold gas surrounding the central engine (e.g. Matsuoka $6 t$ al. 1986). 

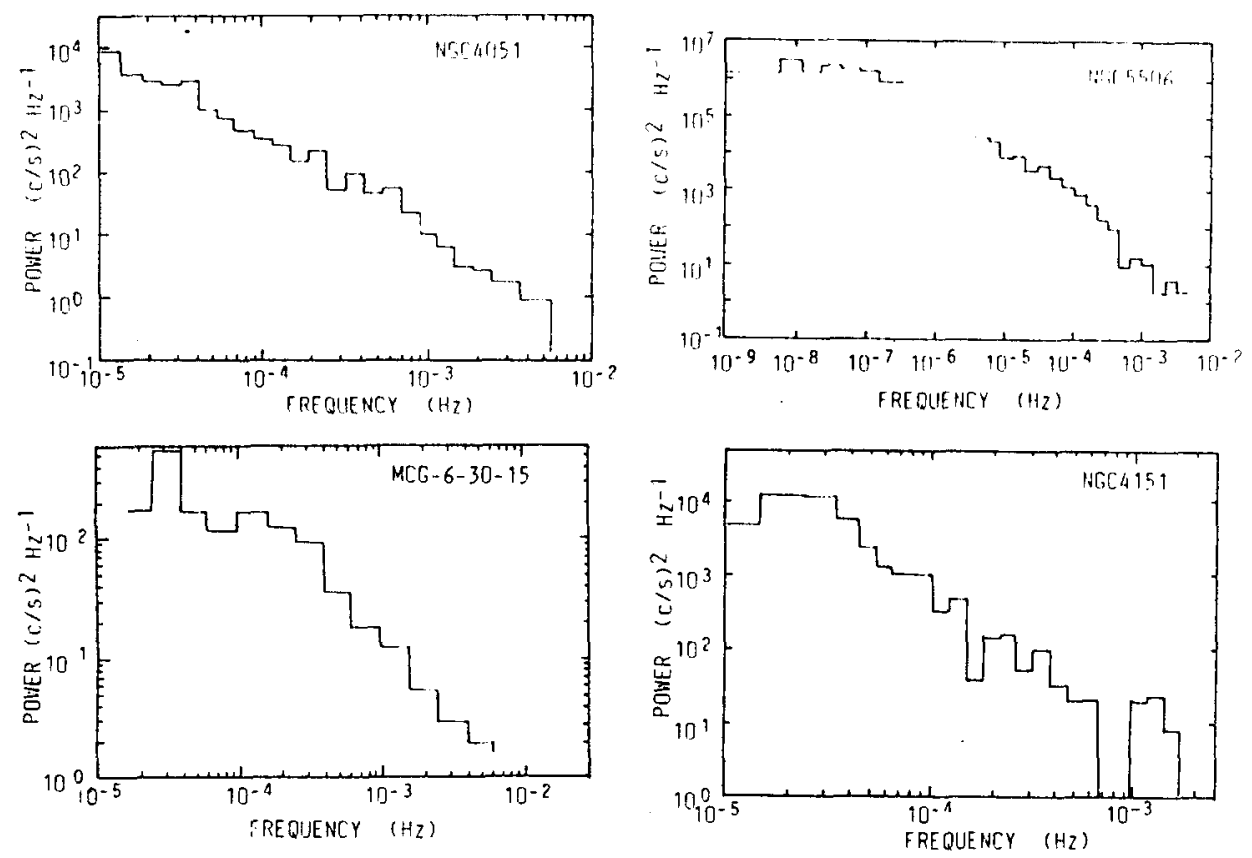

Fig. 3. Power density spectra of X-ray intensities of AGN (Pounds and McHardy 1988), where the power density spectrum of MCG-6-30-15 is obtained from Ginga data by Fiore et al. (private communication, 1991).

Recently Ginga has discussed on exciting phenomenon that the ion $\mathrm{K}$ X-ray flux of NGC6814 correlates with the continuum X-ray (Kunieda $t$ al. 1990; Kunieda et al. 1991). The continuum and iron line intensities observed by Ginga are shown in Fig. 4. This suggests that the fluorescence material should be located at somewhat nearby region from the central engine. Thus the cross correlation analysis has been done for the X-ray line and continuum intensities (Done et al. 1992). Fig. 5 shows a time lag of iron line against the continuum (3-5.5 keV) for NGC6814 (Done $t$ t al. 1992). The lag time was about less than $200 \mathrm{sec}$. This suggests that the fluorescent matter is located at the region less than $10^{13} \mathrm{~cm}$ from the central engine. Such matter would be highly ionized by strong irradiation from the central source (Hayakawa 1991; Yamauchi et al. 1992).

\subsection{Reflection Spectrum}

I would like to mention the reprocessed region with very thick gas (dense matter) derived from recent spectral analyses of X-rays. Prior to Ginga observations, X-ray spectra of AGN had been described by the model of a single power law plus an iron line emission (e.g. Mushotzky 1984; Turner and Pounds 1989). However, detailed observations of the X-ray spectra of 


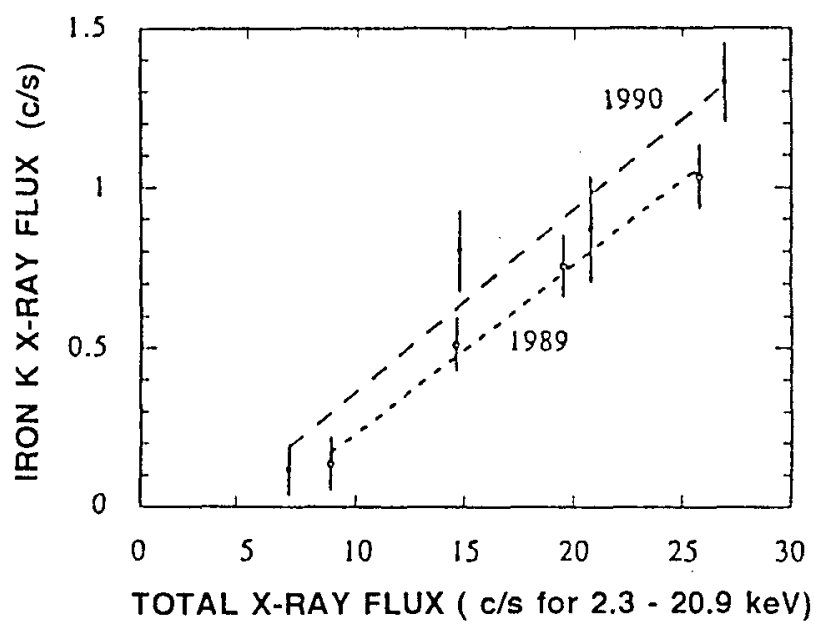

Fig. 4. The X-ray continuum and iron line intensities of NGC6814 (Kunieda et al. 1991).

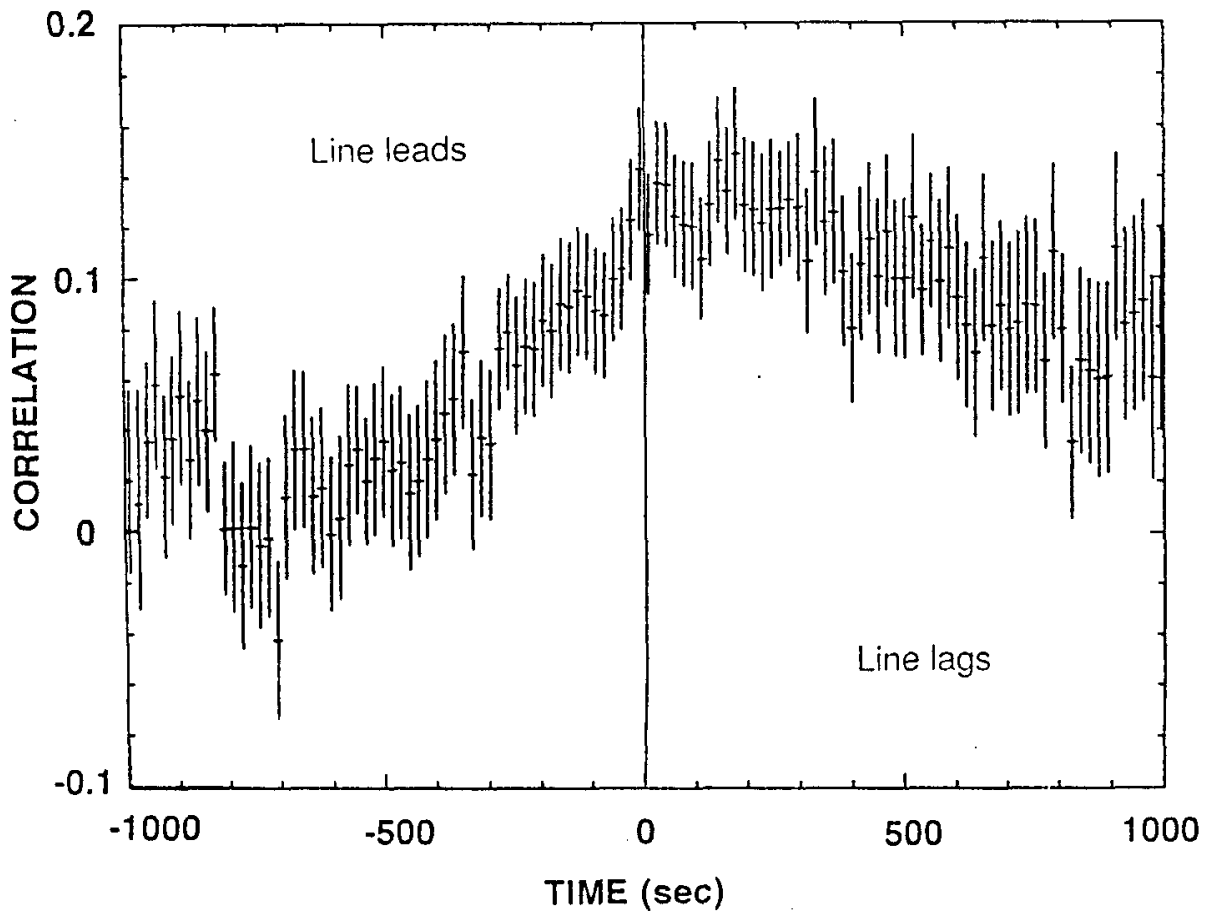

Fig. 5. The cross correlation of the extracted iron $\mathrm{K} \mathrm{X}$-ray and $3-5.5 \mathrm{keV}$ flux (Done $\epsilon t$ al. 1992).

Seyfert galaxies by Ginga have ruled out this model generally (Matsuoka $\epsilon t$ al. 1990; Piro $t$ t al. 1990). As aforementioned, some X-ray spectra require the reflection component in addition to a single power law plus an emission 


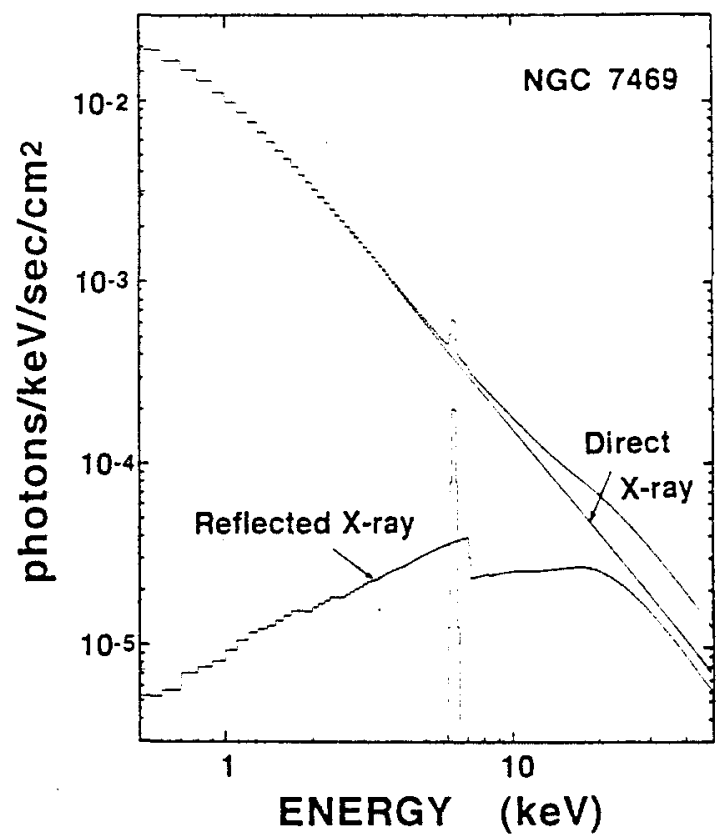

Fig. 6. A reflection model of AGN spectrum; an example of NGC7469.

line.

Detailed observations of the X-ray spectra of AGN have proved that the X-ray spectra of many AGN are well fitted to so called the "reflection model", which consists of direct and reflection components as shown in Fig. 6. This is a leading model of X-ray spectra of AGN, and then this reflection feature would be related to the UV bump; that is, the X-rays from the central engine are reflected by some part of accretion disk. Thus so far the $\mathrm{X}$-ray spectra of a number of AGN observed by Ginga have been well fitted to the reflection model (Seyfert galaxies: Matsuoka et al. 1990; Pounds et al. 1990; Piro et al. 1990; Nandra et al. 1991, QSO's: Williams et al. 1992).

\subsection{VARIABILITY OF SPECTRAL INDEX}

The power law index in AGN X-ray spectra has been called as a "canonical" index (Mushotzky 1984). If the spectra are fitted to a single power law model, the canonical index is represented to be about 1.7 in photon index. But some AGN X-ray spectra show that the power law index depends on the X-ray intensity, if the spectra are fitted to a simple power law model (Halpern 198.5; Perola et al. 1986; Turner 1987; Matsuoka t al. 1990). However, actually the X-ray spectra of many Seyfert galaxies are well fitted to the reflection model. If the reflection model is applied to X-ray spectra of some AGN, it 

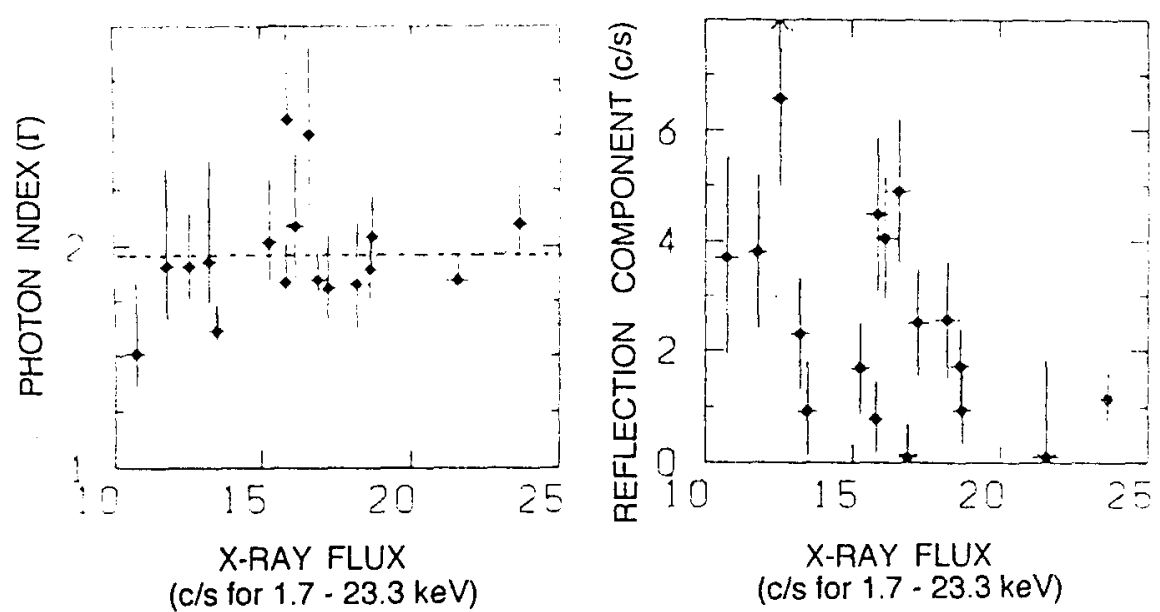

Fig. 7. The photon-index and reflection component for MCG-6-30-15 (Fiore et al. 1992).

seems that the photon index is not variable for the X-ray intensity, while the reflection component is variable for the X-ray intensity as shown in Fig. 7 (Fiore et al. 1992). If we use a single power law model for NGC5548, the photon index is variable for the $\mathrm{X}$-ray flux, while the photon index is constant for the reflection model (Nandra et al. 1991). This is not common to AGN $\mathrm{X}$-ray spectra. In case of NGC4151 the results of Ginga observations indicate the variation in the spectral index (Yaqoob and Warwick 1991; Yaqoob et al. 1992), in which the spectral softening as the source brightens is due to a correlation of the spectral index with $2-10 \mathrm{keV}$ flux as shown in Fig. 8 . Furthermore, it is noted that the X-ray spectrum of NGC4151 is well fitted by a partial covering model, but rules out the generally favoured reflection model (Yamauchi et al. 1992).

The flux vs. index correlation in NGC4151 is interpreted in terms of the current "pair model" of X-ray emission in AGN which involves inverse Compton scattering of UV photons on relativistic electrons (Yaqoob 1992; also see Lightman and Zdziarski 1987).

\subsection{SOFT EXCESS}

Soft X-ray spectra of Seyfert galaxies are complex in form and that contributions from several different emission and absorption components make up the soft spectra (Turner et al. 1991; Urry et al. 1989). Recent AGN survey by ROSAT shows that the soft excess below about $1 \mathrm{keV}$ is common to AGN spectra (Brinkmann 1991). Past observations suggest that at least four types of soft excess exist in Seyfert galaxies as follows (Turner $t$ t al. 1991).

(1) A steep, ultrasoft, and rapidly variable excess, which may be associ- 


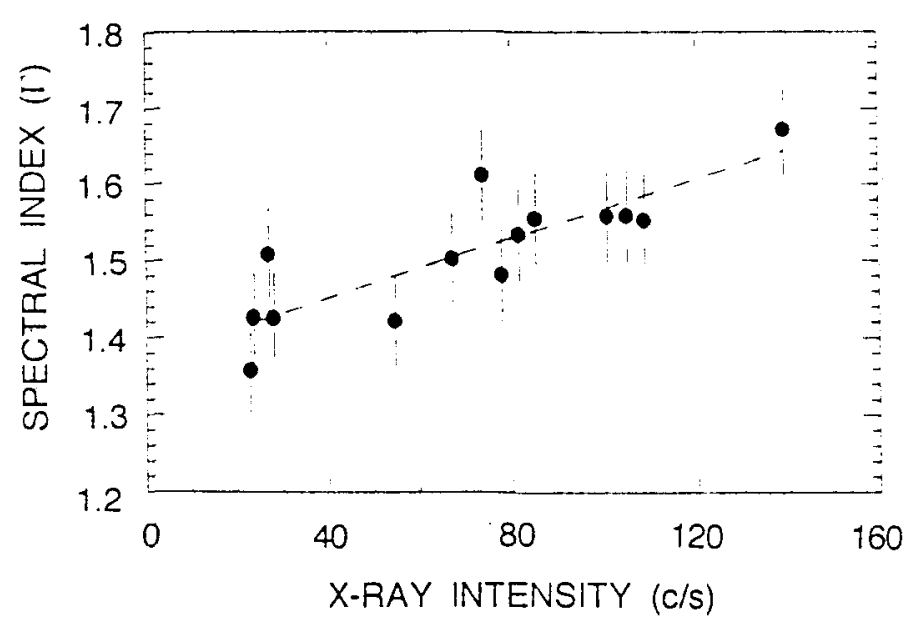

Fig. 8. The photo-index - intensity relation of NGC4151 (Yaqoob et al. 1992).

ated with the inner region of an accretion disk (see Wilkes and Elvis 1987, Turner and Pounds 1989). This component has been characterized as a lowtemperature $(T \sim 6-10 \mathrm{eV})$ black body or thermal bremsstrahlung emission or a a steep second-power law. Most AGN spectra observed by ROSAT seem to be addressed to this type.

(2) Excess soft emission due to leakage of the hard X-ray continuum through a patchy absorber, i.e., the "partially covered" sources. NGC4151 is a typical example of this type (Holt $\epsilon t$ al. 1980; Yamauchi $\epsilon t$ al. 1992).

(3) An emission-line feature typically centered around $0.8 \mathrm{keV}$, whose possible explanation is a blend of soft X-ray lines.

(4) An extended soft excess component seen in Einstein HRI observations of NGC4151 (Elvis $\epsilon t$ al. 1983), and NGC2992 and NGC1566 (Fassnacht $\epsilon t$ al. 1990). This type of soft excess would not be variable in a short time scale.

Detailed observations of Seyfert galaxy NGC5506 were made by Ginga (Bond et al. 1992). Fig. 9 shows a spectral variability of NGC5506 over a full observation period of July 6-10, 1991 and on July 9, 1991. The soft (1.2$3 \mathrm{keV})$, intermediate (3-10 keV), and hard (10-28 keV) X-ray intensities are plotted for each orbit along with the softness and hardness ratio. This $\mathrm{X}$-ray spectrum is not well fitted to a "reflection" model, remaining a soft excess. After investigation of several spectral models, we have achieved the composite model consisting of reflection and partial covering components in addition to the direct one as shown in Fig. 10. Thus this is a generalized model, in which the partial covering model and the reflection model are combined.

The spectra of NGC4151 as well as NGC5506 require the partial covering components. The results of a covering fraction derived from the spectra of these AGN give that the covering fractions normally have a value of 80 - 

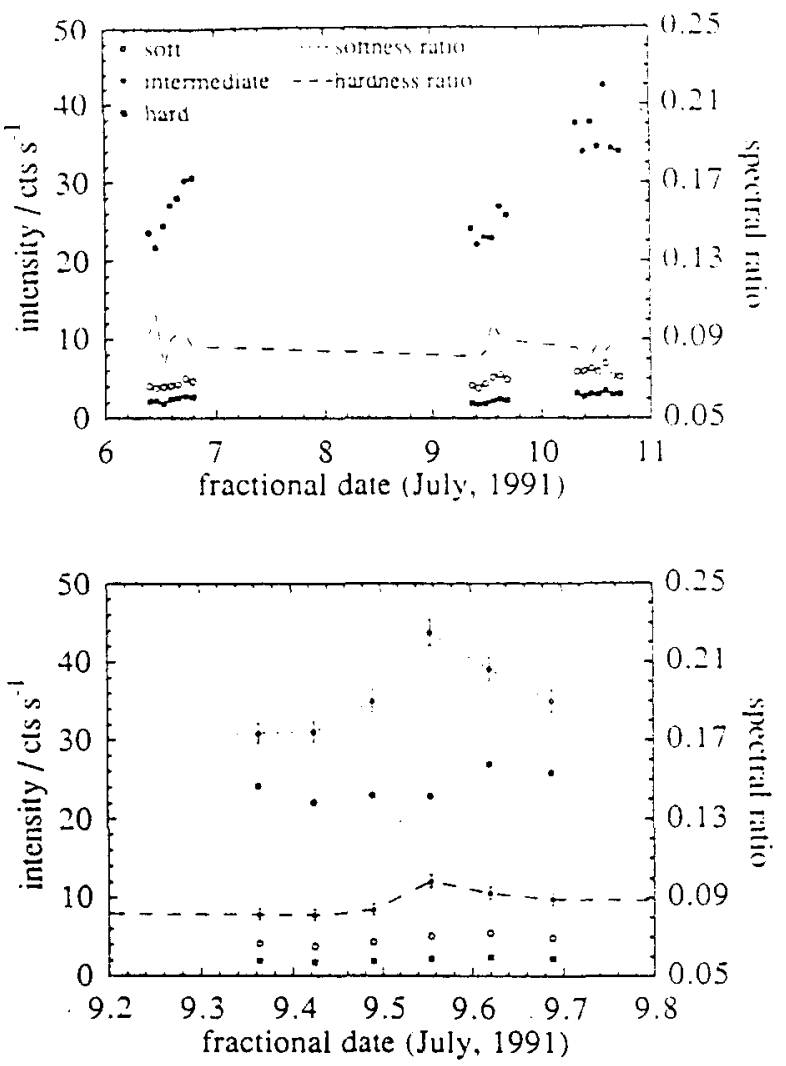

Fig. 9. A spectral variability of NGC5506 (Bond et al. 1992). The soft (1.2-3 keV), intermediate (3-10 keV), and hard (10-28 keV) X-ray intensities are plotted for each orbit along with the softness and hardness ratios.

$90 \%$, but one time declined to around $60 \%$ and $20 \%$ for NGC5506 and NGC4151, respectively, as shown in Fig. 11. The existence of a "canonical" value of the covering fraction would rule out the cloud interpretation of the partial covering model since it is hard to realized a situation where a cloud cover can re-configure itself to a normal state after some distribution such as a temporary break in the clouds (Bond $\epsilon$ al. 1992; laqoob $\epsilon t$ al. 1992). Anyway very dense matter in the central engine of AGN have an important effect on the emission and absorption of the radiation (Celotti $\epsilon t$ al. 1992).

\subsection{Rapid Variabilities of Two AGN}

Ginga detected a surprising flare from the QSO PKS0558-504, whose X-ray flux increased by $67 \%$ in the space of only 3 minutes (Remillard $\epsilon t$ al. 1991). This flare is one of the most dramatic examples of rapid variabilities from AGN. The X-ray rise of PKS0558-504 implies $\mathrm{dL} / \mathrm{dt} \sim 3.2 \times 10^{42} \mathrm{erg} / \mathrm{sec}^{2}$. 

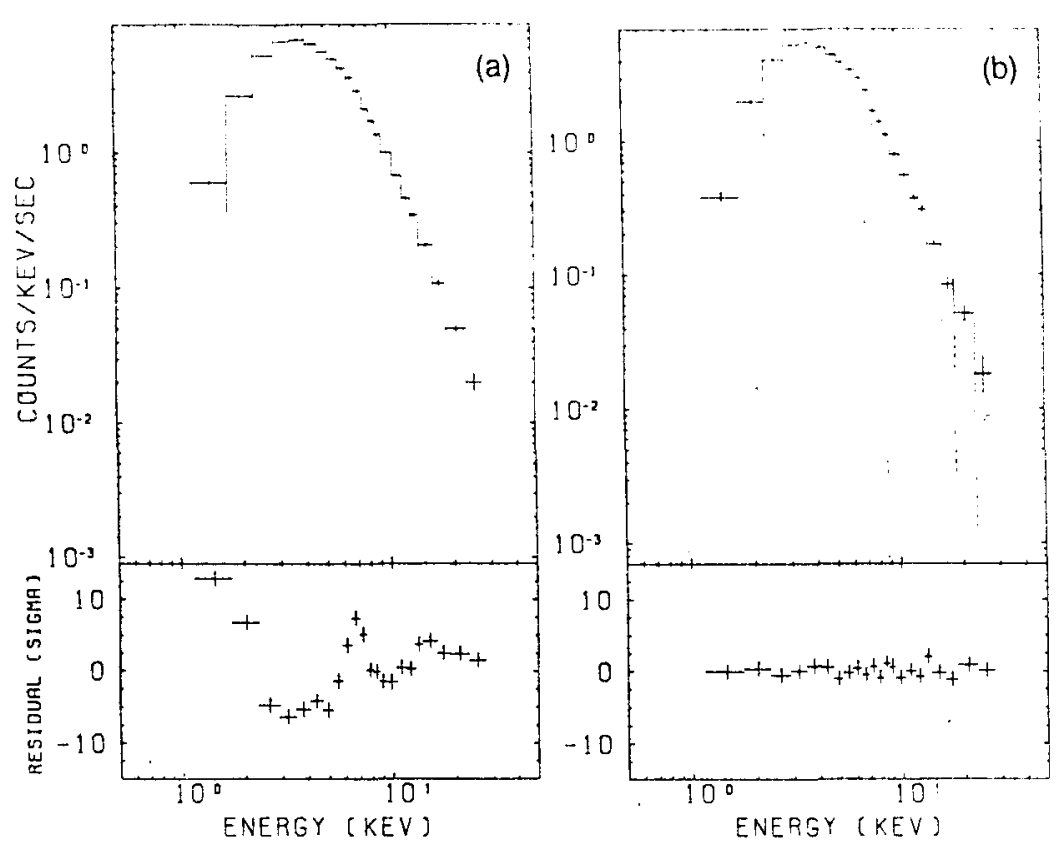

Fig. 10. The NGC5506 spectral fits are shown for two cases of a single power law model and a composite model consisting of "reflection" and "partial covering" models (Bond $\epsilon t$ al. 1992).
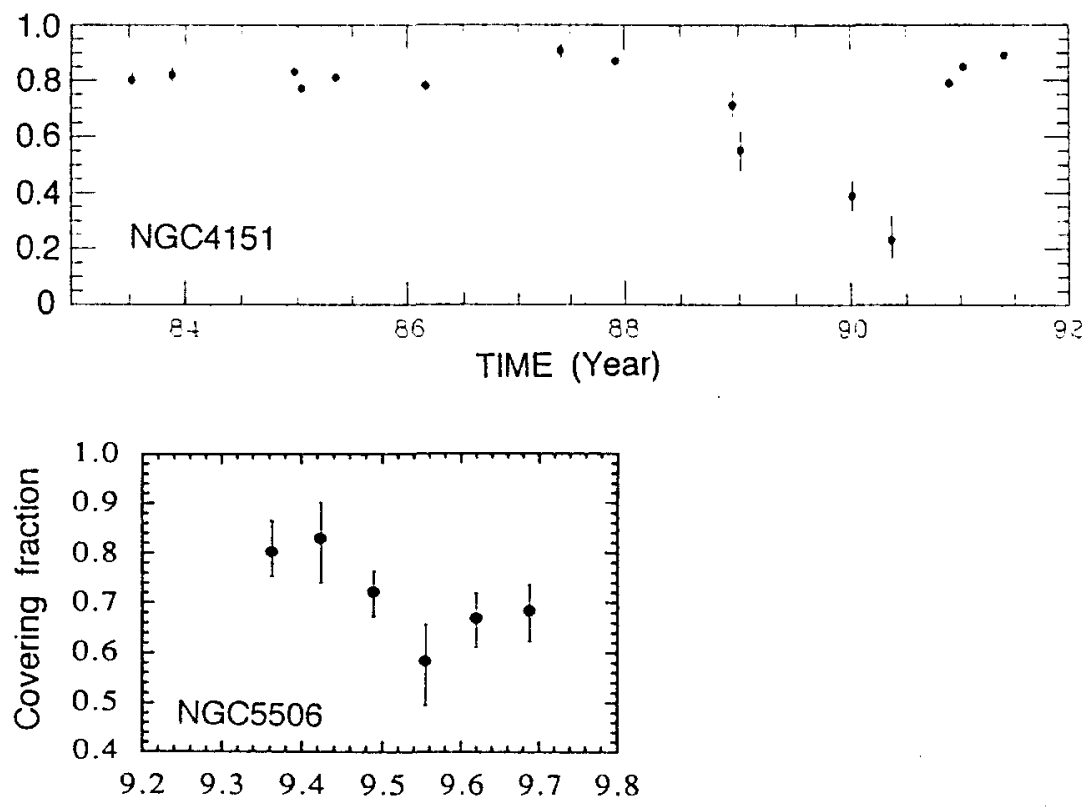

Fractional date (July, 1991)

Fig. 11. Time variabilities of covering fractions for NGC5506 (Bond et al. 1992) and NGC4151 (Yaqoob et al. 1992). 


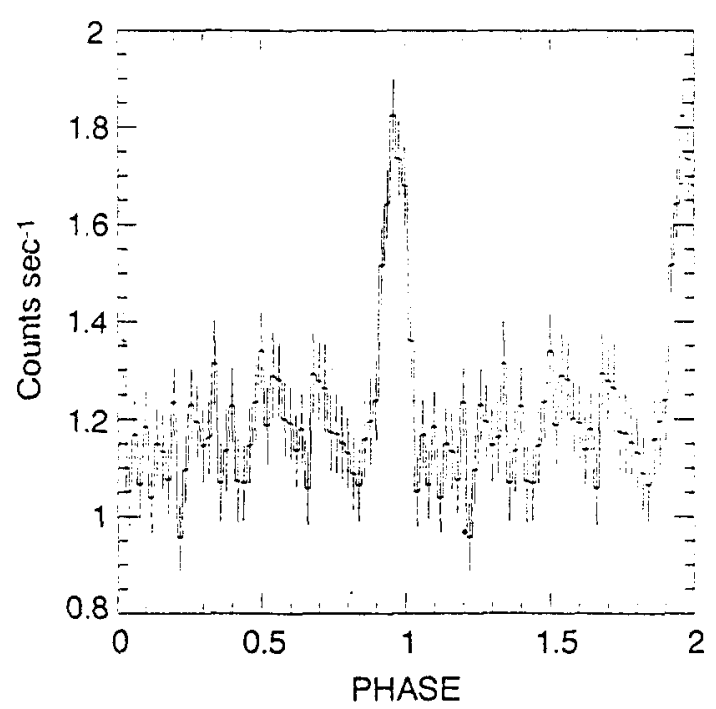

Fig. 12. A light curve folded on the period of $12,200 \mathrm{sec}$ for NGC6814 X-ray light curve (Fiore et al. 1991).

Ginga also detected that another QSO 3C279 exhibited a $20 \%$ increase in 45 minutes giving a change in luminosity of $2-10 \mathrm{keV}, \mathrm{dL} / \mathrm{dt}>2.9 \times 10^{42}$ $\mathrm{erg} / \mathrm{sec}^{2}$ (Makino tt al. 1990 ).

In the interpretation of AGN variability time scales an efficiency limit $(\eta)$ is derived for the rate of conversion of gravitational potential energy into X-ray emission, assuming that the photons must escape through the accreting matter, and that the photon opacity is dominated by Thomson scattering. The derived limit is $\mathrm{dL} / \mathrm{dt}<2 \times 10^{42} \eta \mathrm{erg} / \mathrm{sec}^{2}$ in a spherical symmetric model (Cavallo and Rees 1978; Fabian 1979). It is unlikely that $\eta$ exceeds 0.1 . The results of both PKS0558-504 and 3C279 suggest that the apparent luminosity must be enhanced by relativistic beaming (Remillard et al. 1991). Such as rapid variabilities in X-ray luminosity have been attained by BL Lac objects (Barr and Mushotzky 1986).

Further surprising result of a periodicity of $A G N$ is discovered in Seyfert galaxy, NGC6814, on a time-scale of approximate $12,000 \mathrm{sec}$ during long observations by EXOSAT (Mittaz and Branduardi-Raymont 1989; Fiore et al. 1992) as a light curve folded on the period of 12,200 sec shown in Fig. 12. It has been interpreted that a bright spot is moving surrounding the central object (Abramowicz $t$ t al. 1992), or some peculiar dynamic oscillation of the disk (Honma $\epsilon t$ al. 1992) and so on, but the further explanation is open to question. 


\section{Conclusion}

Recent results of X-ray time variabilities as well as X-ray spectra have made a great progress for a structure of a nearby region of their central engine. The reprocessed region as well as direct $X$-ray region has an important role in $A G N$. Thus we could inspect most nearest region in AGN by X-ray observations. For further future observations with ASTRO-D and SPECTRUM-X it is important to investigate the variability of $\mathrm{X}$-ray intensity in connection with the X-ray spectral structure. Especially, the observation of the energy shift of X-ray lines is one of the most important subjects. Systematic investigation of intrinsic absorption column densities and soft excesses in X-rays will also provide a great progress for AGN.

\section{References}

Abramowicz, M. A., Bao, G., Fiore, F. et al.: 1992, Nature, submitted.

Antonucci, R. R. J. and Miller, J. S.: 1985, Astrophysical Journal 297, 621.

Awaki, H. et al.: 1990, Nature 34, 544 .

Barr, P. and Mushotzky, R. F.: 1986, Nature 320, 421.

Begelman, M. C. and De Kool, M. 1991, Variability of active galactic nuclei, eds. H. R. Miller and P. J. Wiita (Cambridge Univ. Press.), p. 198.

Bond, I. A., Matsuoka, M. and Yamauchi, M.: 1992, Astrophysical Journal, submitted.

Brinkmann, W.: 1991, MPE conference

Cavalo, G. and Rees, M. J.: 1978, Monthly Notices of the RAS 183, 359.

Celloti, A., Fabian, A. C. and Rees, M. J.: 1992, Monthly Notices of the RAS 255, 419.

Done, C., Madejski, G. M., Mushotzky, R. F., Turner, T. J. Koyama, K., and Kunieda, H. 1992, Frontiers of X-ray Astronomy, eds. Y. Tanaka and K. Koyama (Univ. Academy Press), p. 525.

Elvis, M., Briel, U. G. and Henry, J. P.: 1983, Astrophysical Journal 268, 105.

Fabian, A. C.: 1979, Proc. Roy. Soc. 336, 449.

Fabian, A. C., George, I., Miyoshi, S. and Rees, M. J.: 1990, Monthly Notices of the RAS $242,14$.

Fassnacht, C., Elvis, M., Wilson, A. S. and Briel, U.: 1990, Astrophysical Journal 361, 459.

Fiore, F., Massaro, E. and Barone, P.: 1991, Astronomy and Astrophysics, submitted.

Fiore, F., Perola, G. C., Matsuoka, M., Yamauchi, M. and Piro, L. :1991, Variability of Active Galactic Nuclei, eds. H. R. Miller and P. J. Wiita (Cambridge Univ. Press), p. 273.

Fiore, F., Perola, G. C., Matsuoka, M., Yamauchi, M. and Piro, L. : 1992, Astronomy and Astrophysics, to be appeared.

Guilbert, P. W. and Rees, M. J.: 1988, Monthly Notices of the RAS 233, 475.

Halpern. J. P.: 1985, Astrophysical Journal 290, 130.

Hayakawa, S.: 1991, Nature 351, 214.

Holt, S. S., Mushotzky, R. F., Becker, R. H., Boldt, E. A., Serlemitsos. P. J., Szymkowiak, A. E. and White, N. E.: 1980, Astrophysical Journal, Letters to the Editor 241, L13.

Honma, F., Matsumoto, R. and Kato, S.: 1992, preprint.

Lighman, A. J. and White, T. R.: 1988, Astrophysical Journal 335, 57.

Lightman, A. P. and Zdziarski, A. A.: 1987, Astrophysical Journal 319, 643.

Kawara, K., Nishida, M. and Gregory, B.: 1989, Nature 341, 27.

Kawara, K., Nishida, M. and Gregory, B.: 1990, Astrophysical Journal 352, 433.

Koyama, K., Inoue, H., Tanaka, Y., Ohashi, T. and Matsuoka, M.: 1989. Publications of the ASJ 41, 731 . 
Kuieda. H. et al: 1990. Nature 235, 786.

Kunieda, H. et al: 1991, Iron Line Diannostics in A'ray Sources. eds. A. Treves, G. C. Perola and L. Stella (Spring-Verlarg), p. 241.

Malkan, M. A. and Sargent. W. L. W.: 1982, Astrophysical Journal 254, 22.

Makino. F. et al.: 1990, Astrophysical Journal, Letters to the Editor 347, L9.

Matsuoka, M., Ikegami, T., Inoue, H. and Koyama, K.: 1986, Publications of the ASJ 38, 28.5 .

Matauoka, M., Piro, L., Yamauchi, M. and Murakami. T.: 1990, Astrophysical Journal 361,440 .

McHardy, J. M. 1989. 29rd ESL.AB Symposium X-ray Astronomy, 2. AG.N and the X-Ray Background, eds. by J. Hunt and B. Battrick (ESA-France), p.111.

Mittaz, J. P. D. and Branduardi-Raymont, G.: 1989. Monthly Notices of the RAS 238, 1029.

Mulchaey, J. S., Mushotzky, R. F. and Weaver, K. A.: 1992, Astrophysical JournalLetters, in press.

Mushotzky, R. F.: 1984, Adv. Space Res. 3, No. 10-12, p.153.

Nandra, K., Pounds, K. A. et al.: 1991, Monthly Notices of the RAS 248, 760.

Netzer, H.: 1990, "Active Galactic Nuclei" eds. by R. D. Blaudford, H. Netzer and L. Woltjer (Springer-Verlag), p.57.

Piro, L. Yamauchi, M. and Matsuoka, M.: 1990, Astrophysical Journal, Letters to the Editor 360, L35.

Pounds, K. A. and McHardy, I. M.: 1988, Physics of Neutron Stars and Black Holes, Ed. by Y. Tanaka (Universal Academy Press. Inc.), p. 285.

Pounds, K. A., Nandra, K., Stewart, G. C., George, I. M. and Fabian, A. C.: 1990, Nature $344,132$.

Remillard, R. A. et al.: 1991, Nature 350, 589.

Sanders, D. B., Phinnery, E. S., Neugebauer, G., Soifer, B. T. and Mathews, K.: 1989, Astrophysical Journal 347, 29.

Sun, H-W. and Malkan, M. A.: 1989, Astrophysical Journal 346, 68.

Turner, T. J., Weaver, K. A., Mushotzky, R. F., Holt, S. S. and Madejski, G. M.: 1991, Astrophysical Journal 381, 85.

Turner, T. J. and Pounds, K. A.: 1989, Monthly Notices of the RAS 240, 833.

Erry, C. M., Arnaud, K. A., Edelson, R. A., Kruper, J. S. and Mushotzky, R. F.: 1989. Proceedings of the 23rd ESLAB Symp., eds. J. Hunt and B. Battrick (ESA publications), p. 789 .

Wilkes, B. and Elvis, M.: 1987, Astrophysical Journal 323, 243.

Williams, O. R., Turner, M. J. L., Stewart, G. C. et al.: 1992, Astrophysical Journal, in press.

Yamauchi, M., Matsuoka, M., Kawai, N. and Yoshida, A.: 1991, Astrophysical Journal, in press.

Yaqoob, T.: 1992, Monthly Notices of the RAS in press (March 1992).

Yaqoob, T. et al.: 1992, in preparation.

Yaqoob, T. and Warwick, R. S.: 1991, Monthly Notices of the RAS 248, 773. 